\author{
MAHENDRA SINGH \\ Uttar Pradesh State Obscrvatory Manora Peak \\ Naini Tal- 263 129, India \\ MASAHIRO MON and TOMOKAZU KOGURE \\ Department of Astronomy, Faculty of Science \\ Kyoto University Sakyo-Ku Kyoto 606, Japan \\ MASAKAZU SUZUKI \\ Kanazawa Institute of Technology \\ P.O.Kanazawa-South,Ishikawa 921 Kanazawa,Japan
}

\begin{abstract}
Based on a series of high dispersion Coude spectra taken with 188-cm telescope of Okayama Astrophysical Observatory over an extensive period of time ( nearly 20 years),spectroscopic behaviours were measured and discussed. Synchronous rariations of $V / R$ (intensity ratio of riolet to red emission peaks) variability with the radial velocity curves (RV) for Balmer and HeI 3888 shell absorption lines were confirmed. Using the peak separations of the Balmer emission lines, envelope extension was also estimated. Main difficulty was found in explaining the envelope extension of the primary star of the system which orerfow's the Roche-lobe limit when we consider the binary nature of the system.
\end{abstract}

\title{
INTRODUCTION
}

Phi-Per is a well known spectroscopic binary Be Shell star with the spectral type B2 IV ep (Lesh, 1968).Radial velocity variations of this star were first noted by Campbell(1902) and were attributed to binarf motion by Lundendorff (1910) and Cannon (1910). Lockyer $(1925,1926)$ was the first to notice its $V / R$ variability synchronous with phase.Poeckert (1981) and Suruki (1980) have derived the orbital parameters of the system and found that the masses of the systemic components are $20 M_{\odot}$ and $4 M_{\odot}$ with the separation $1.42 \mathrm{AU}$.

In this paper we present our new spectroscopic observations (Coude spectra) taken with 188-cm telescope of Okasama Astrophysical Observatory. The dispersion nas $10 \mathrm{~A} / \mathrm{mm}$. All the observational data nere folded for the orbital period of 126.696 days with the epoch $T_{0}=J D(H e l) 2424473.500$ (Hickok,1969).

The radial velocity $(R V)$ and $V / R$ (intensity ratio of violet to red emission components) variability for the Balmer and HeI 3888 shell absorption lines were determined. Using the peak sepatations of emission components of Balmer lines the extension of the envelope was estimated.

\section{OBSERVATIONS and MEASUREMENTS}

Nearly 100 Coude spectrograms taken with 188-cm reflector of Okayama Astrophysical Observatory (1968-1986) were analysed. PDS(PE) microdensitometer measurements were done at $K$ wasan Observatory, Kyoto University. The data were processed at the Computer Centre of the Kanazawa Institute of Technology and Kyoto with the help of a software developed by one of our authors M.Suzuki. University. Computer plots of intensity curve in units of both the radial velocity and wavelengths were obtained for individual spectra. From these plots RV values at different points of the line profiles 
were measured. Correspondingly, intensity values for the emission components were also measured to study the $V / R$ variability. The $R V$ curve for the Balmer $\left(H_{\beta}, H_{\gamma}, H_{6}\right)$ and $\mathrm{HeI} 3888$ shell lines are shown in Figures 1 and 2. $A$ plot of $\log V / R$ against phase is shown in Fig 3.The peak separations was varying between 150 to $250 \mathrm{~km} / \mathrm{s}$. The envelope extension $R_{e}$ was determined using $R_{e}=4 G M_{1} / V_{p}^{2}$ (Poeckert, 1981), where $M_{1}$ is the mass of the primary and $V_{p}$ is the peak separation of the emission line.

\section{RESULTS and DISCUSSIONS}

From Figs. 1,2 and 3 ,it is clear that the radial velocity and $V / R$ both vary with phase synchronously. There is no phase lag. Occasionally this star exhibits quadrupole peaks with two peak separations (Kogure et. al. 1991) suggesting some complicated structure of the envelope which can be interpreted in terms of a double ring structure. If we calculate the envelope extension using Poeckert(1981) expression, we find that the envelope extends upto $265 R_{\odot}$ for a peak separation of $200 \mathrm{~km} / \mathrm{sec}$ and goes beyond the Roche-lobe of the primary star of the system.

\section{REFERENCES}

Campbell, W.W. 1902,Astrophys.J.16,195.

Cannon, J.B. 1910,J.R.A.S., Canada 4,195.

Hickok, F.R. 1969,M.Sc.Thesis, university of Toronto, Canada.

Kogure, T.,Mon, M.,Suzuki, M.,Singh, M.1991,

Pac.Rim Colloq.on New Frontiers in Binary Stars Research

held on Nov 5-13,1990 at Seoul and Taejon, Korea(in press).

Lesh, J.R. 1968, Astrophys. J.Suppl. 17,371 .

Lockyer, W.J.S. 1925, M.N.R.A.S. 85,580.

Lockyer, W.J.S., 1926, M.N.R.A.S. 86,474.

Lundendorff, H.1910, Astr.Nachr186,17.

Poeckert, R., 1981,P.A.S.Pacific, 03,297.

Suzuki, M., 1980, Publ.Astron.Soc.Japan, 32,331.

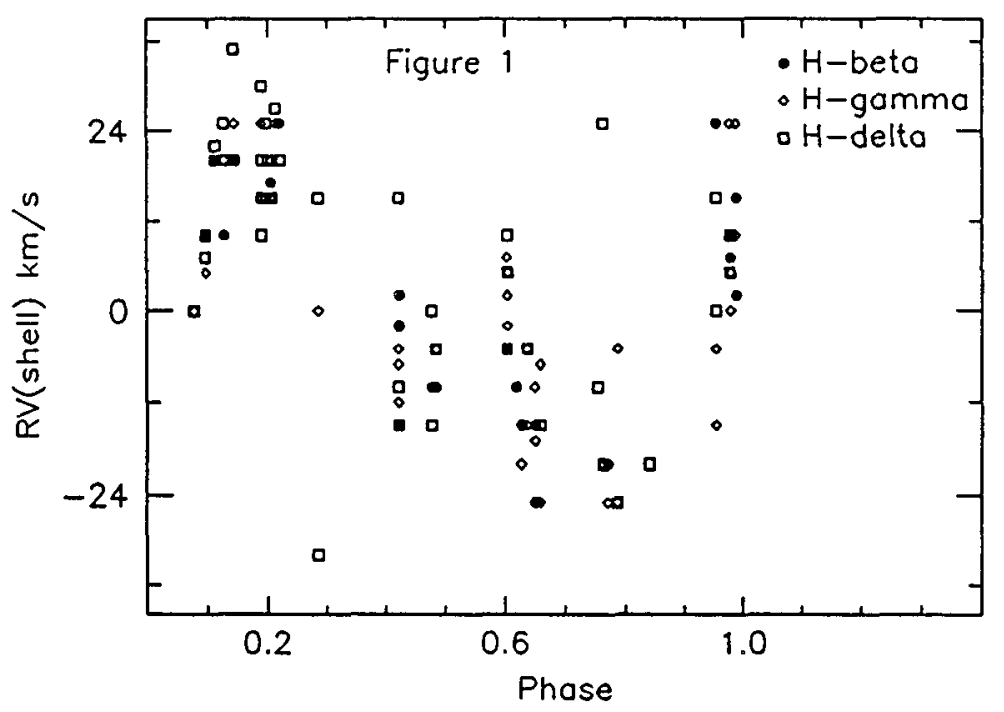



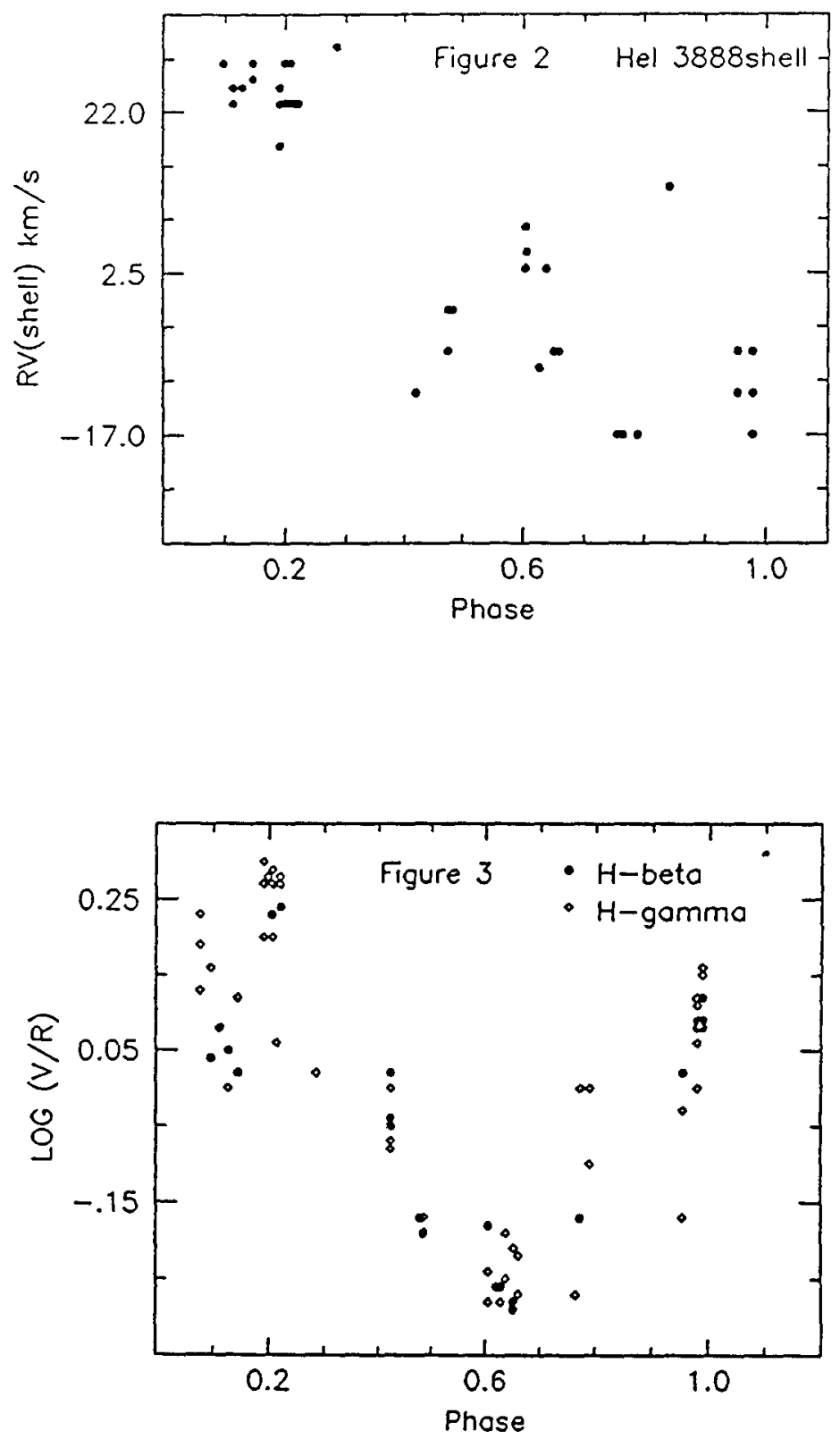